\title{
Fulminant Hepatic Failure Due to Dengue
}

\author{
Sedhain A, Adhikari S, Regmi S, Chaudhari SK, Shah M, Shrestha B
}

Department of Medicine

Chitwan Medical College

Bharatpur, Chitwan Nepal.

CORRESPONDING

Dr. Arun Sedhain

Department of Medicine

Chitwan Medical College

Bharatpur, Chitwan Nepal.

E-mail: arunsedhain@gmail.com

Citation

Sedhain A, Adhikari S, Regmi S, Chaudhari SK, Shah $M$, Shrestha B.Fulminant Hepatic Failure due to Dengue. Kathmandu Univ Med J 2011;34(2):73-5.

\begin{abstract}
:
Dengue Fever (DF) is only rarely considered as a cause of acute liver failure even globally and only a few case reports of acute hepatic failure and encephalopathy occurring in DF in adults are available. We report a case of Acute Liver Failure due to Dengue during a major outbreak in 2010 in Chitwan.

A 20 year old previously healthy female presented to the emergency department of Chitwan Medical College with fever, jaundice and altered sensorium. She was tested positive for Dengue IgM. Her biochemical and clinical parameters were suggestive of acute liver failure with total billirubin of $10.1 \mathrm{mg} / \mathrm{dL}$, direct billirubin of $5.2 \mathrm{mg} /$ dL, ALT 5760 IU, AST 14100 IU, alkaline phosphatase of 1250 IU, PT INR of 1.76 and platelet count of $30,000 / \mathrm{mm}^{3}$. Other causes for acute hepatic failure like acute viral hepatitis, leptospirosis, malaria, Reye's syndrome were ruled out. The patient was admitted and managed in the ICU with supportive care and platelet transfusion. With treatment she made a significant clinical and biochemical improvement with AST of 105 IU, ALT of 120 IU and platelet count of $150,000 / \mathrm{mm} 3$. She was discharged after 11 days of hospital stay.
\end{abstract}

\section{KEY WORDS}

Dengue, Dengue Hemorrhagic Fever, Fuminant Hepatic failure

\section{INTRODUCTION}

Dengue Fever, which is endemic in Southeast Asia, was first recorded in Nepal in 2004. ${ }^{1}$ Then a confirmed outbreak was observed in nine districts of Terai region in Nepal in $2006 .^{2}$ Although dengue had been considered a rare disease in Nepali context, an outbreak of the disease is seen recently in Chitwan and adjacent areas of Nepal. The term acute hepatic failure is used to describe the development of coagulopathy, usually with INR of greater than 1.5, and any degree of mental alteration (encephalopathy) in a patient without preexisting cirrhosis and with an illness of less than 4 weeks. However, Dengue is only rarely considered as a cause of acute liver failure even globally and only a few case reports of acute hepatic failure and encephalopathy occurring in Dengue in adults are available. We, hereby, present a 20 year old female with Dengue fever who presented with clinical and biochemical features of acute hepatic failure.

\section{CASE REPORT}

A 20 year-old previously healthy, non-alcoholic female presented to the Emergency Department of Chitwan
Medical College, Nepal with the history of fever and headache for seven days, nausea and vomiting for 4 days and progressive deterioration in sensorium with altered sleep cycle and restlessness for one day. She had no bleeding manifestations. She had a recent travel history to northern area of India. On admission, she was restless and her GCS was $12 / 15$ (E4, V3, M5). She had a axillary temperature of $99.80 \mathrm{~F}$, blood pressure of $90 / 60 \mathrm{mmHg}$, respiratory rate of $20 / \mathrm{min}$ and Pulse rate of $98 / \mathrm{min}$. She had icterus. Respiratory and Cardiovascular system examination was normal. Abdominal examination revealed hepatomegaly with a liver span of $13 \mathrm{cms}$. There was no free fluid in the abdominal cavity. Bilateral plantar responses were withdrawal. There were no signs of meningeal irritation.

\section{Laboratory Investigations}

Hemogram revealed an $\mathrm{Hb}$ of $10.5 \mathrm{gm} \%$, TLC $8900 / \mathrm{mm}^{3}$, and Platelet count $30,000 / \mathrm{mm}^{3}$. Her urea was 80 and creatinine of $2.0 \mathrm{mg} / \mathrm{dL}$, serum $\mathrm{Na}^{+}$was $126 \mathrm{mmol} / \mathrm{L}$, and serum $\mathrm{K}^{+}$of $4.1 \mathrm{mmol} / \mathrm{L}$. Liver function test showed total billirubin of $10.1 \mathrm{mg} / \mathrm{dL}$ with direct billirubin of $5.2 \mathrm{mg} /$ dL, ALT 5760 IU, AST 14100 IU and alkaline phosphatase of 
1250 IU. Prothrombin time was deranged with INR of 1.76. In view of recent outbreak of dengue and leptospirosis in our area, serology for dengue, leptospira and malaria was sent. Her Dengue serology for IgM was positive but serology for leptospira and malaria were negative. Ultrasound of the abdomen revealed hepatomegaly, minimal pleural effusion and minimal ascites and distended gall bladder. In view of deranged liver function and altered sensorium, a working diagnosis of acute liver failure was made. The patient was fluid resuscitated and shifted to intensive care unit where she was symptomatically managed with IV fluids, antiemetics, platelet transfusion and close monitoring. There were no bleeding episodes. Despite her gradually progressive renal biochemical impairment (maximum creatinine of $3.4 \mathrm{mg} / \mathrm{dL}$ ), she maintained good urine output during the hospital stay. In view of significant thrombocytopenia, she received total of 6 units of platelet rich plasma (PRP). She was also given a course of broad spectrum antibiotic to prevent secondary bacterial infection. Her general condition gradually improved. She started talking normally, icterus gradually decreased, fever and vomiting subsided. Detailed work up for the possible cause for acute liver failure was done. HBsAg and Antibody to hepatitis C, A and E were negative. Hence a final diagnosis of acute liver failure secondary to primary dengue infection was made. With significant clinical and biochemical improvement (Total billirubin: $6.1 \mathrm{mg} / \mathrm{dL}, \mathrm{ALT}$ : 630 IU/L, AST: 993 IU/L, platelet count: 70,000/mm3 and Serum creatinine of $1.8 \mathrm{mg} / \mathrm{dL}$ ), she was shifted to general ward on day 7. She was discharged from the hospital after 11 days of total hospital stay. At the time of discharge her AST and ALT value were $105 \mathrm{IU} / \mathrm{L}$ and $120 \mathrm{IU} / \mathrm{L}$ respectively.

\section{DISCUSSION}

Dengue infections are caused by a flavivirus which has four serotypes (DEN 1-4). It is the commonest arbovirus and a common cause of hemorrhagic fever in the world. The clinical presentation of the disease ranges from simple undifferentiated viral fever to Dengue Hemorrhagic fever and Dengue Shock Syndrome.

In recent times, the reports of rare clinical manifestations of Dengue such as encephalitis, myocarditis, Guillian Barre syndrome, hemolytic uremic syndrome and hepatic manifestations have become more common., ${ }^{3,4}$ Hepatic injury with Dengue infections has been described since 1967 and acute liver failure in association with dengue was initially reported during the epidemic in Indonesia in the 1970s. ${ }^{5}$ After this several cases of fulminant hepatitis with high mortality have been reported. ${ }^{6}$

The term acute hepatic failure is used to describe the development of coagulopathy, usually with INR of greater than 1.5, and any degree of mental alteration (encephalopathy) in a patient without preexisting cirrhosis and with an illness of less than 4 weeks. ${ }^{7}$ Although liver is not the main target organ, direct infection of hepatocytes and Kupffer cells by dengue virus can be observed. ${ }^{8}$ An increase in liver transaminases is observed in the first week of dengue infection mainly in dengue hemorrhagic fever rather than in dengue fever. This can vary from 2-3 folds to more than 10 fold rise from normal level. SGOT rises more than SGPT which is different from other types of viral hepatitis. $^{8}$

The clinical and biochemical presentation in our case was like that of acute liver failure. The important differential diagnosis in a case presenting with fever and acute hepatic failure includes acute viral hepatitis, complicated malaria, leptospirosis, and Reye's syndrome. Negative viral markers for all viral hepatitis ruled out the possibility of viral hepatitis. Negative serology for malaria ruled out the possibility of malaria. Moreover, there wouldn't be marked elevation of ALT and AST in complicated malaria (ALT and AST values of 5760 and 14100 respectively) as seen in our patient. Negative serology test for leptospirosis was a supportive test to rule out leptospirosis in this patient. Reye's syndrome usually does not present with jaundice. Moreover thrombocytopenia and plasma leakage as seen in our patient are unusual in Reye's syndrome. ${ }^{7}$

The presence of thrombocytopenia, signs of plasma leakage, hypotension and positive IgM serology for Dengue in the context of outbreak of dengue in Chitwan and adjacent districts of Nepal since August 2010 strongly suggest the possibility of Dengue shock syndrome (DSS) with acute liver failure in this case.

Acute hepatic failure due to dengue infection with subsequent complete recovery has been reported in two adult patients from India, one from Singapore, one from USA and one from Sri Lanka. ${ }^{9-12}$ However in none of the reports, the liver enzymes were as high as that seen in our patient. Despite the clinical presentation of acute hepatic failure with up to 500 times rise in aminotransferases, our patient had complete recovery.

\section{REFERENCES}

1. Pandey BD, Rai SK, Morita K, Kurane I. First case of dengue virus infection in Nepal. Nepal Med Coll J 2004; 6: 157-9.

2. Pandey BD, Morita K, Khanal SR et al. Dengue virus, Nepal. Emer Infect Dis 2008; 14: 514-5.

3. George R, Lum LCS, Clinical spectrum of dengue infection in Gubler DJ and Kuno G Eds Dengue and Hengue hemorrhagic fever. Washington Cab International, 1997.

4. George R, Liam CK, Chua CT, Lam SK, Pang T, Geethan R, Foo LS. Unusual clinical manifestations of dengue virus infection. Southeast Asian J Trop Med Pub Health 1998; 19 (4): 585-90.

5. Munasinghe DR, Rajasuriya K. Hepatitis in Dengue fever. Ceylon Med J $1967 ; 12(4):$ 222-3.

6. Lawn SD, Tilley R, Lloyd $\mathrm{G}$ et al. Dengue hemorrhagic fever with fulminant hepatic failure in an immigrant returning to Bangladesh. Clinical Infectious Diseases. 2003; 37, e1-e4.

7. Kumar R, Tripathi P, Tripathi S, Kanodiya A, enkatesh V. Prevalence of dengue infection in north Indian children with acute hepatic failure. Annals of Hepatoogy 2008; 7(1):59-62. 
8. Seneviratne SL, Malavige GN, de Silva HJ. Pathogenesis of liver involvement during dengue viral infections (Review). Transactions of the Royal Society of Tropical Medicine and Hygiene 2006; : 608-14.

9. Vinodh BN, Bammigatti C, Kumar A, Mittal V. Dengue fever with acute liver failure. J Postgrad Med 2005; 51:322-3.

10. Subhash $G$, et al. Acute hepatic failure due to dengue: A case report. Cases J 2008; 1: 204.
11. Subramanian V, Shenoy S, Joseph AJ. Dengue haemorrhagic fever and fulminant hepatic failure, Digestive diseases and sciences, 2005 June; 50(6): p1146-7.

12. Gasperino J, et al. Fulminant liver failure secondary to haemorrhagic dengue in an international traveler. Liver Int. 2007 Oct; (8): 1148-51.

13. Wijeweera SCJ, Senevirathna K, Ragunathan MK, A case of fulminant liver failure in dengue haemorrhagic fever. Galle Medical Journal. 2009; 14: (1): 62-63. 